\title{
Digital Culture: Blurred Boundaries and Ethical Considerations
}

\author{
Ben Light \\ School of Media, Music and Performance \\ University of Salford \\ Salford, M3 6EN \\ b.light@salford.ac.uk
}

Steve Sawyer

School of Information Studies

Syracuse University

Syracuse, NY 132244-4100 USA

ssawyer@syr.edu

The Weberian sense of work and life suggests that working is something around which the rest of life flows. Moreover, work life and domestic life have been defined as separate for most people based on physical structures. That is, being physically in a building at work limited your ability to interact with those who are not nearby - not part of work. As such, social conventions regarding the uses of media at work have become part of our cultural sensibilities - we 'know' it is not proper to have romantic discourse over the office phone, much less romance during work! Doing so becomes news. Yet, despite the construction of such distinctions, these work spaces and places have always been difficult to render as such. For example, one might consider the relatively recent development of teleworking from the 1980s or the 'putting out system' ${ }^{1}$ which dates back to the 1400s - both requiring work in the home. The papers in this special issue draw our attention to some of the ethical issues raised by the growing pervasiveness of information and communications technologies (ICTs) in our everyday lives and the fact that it is becoming increasingly difficult to make distinctions between being somewhere (like work) and being away from some things (like one's friends, social interests and other parts of life that are not integrated into this space or place ${ }^{2}$ ).

Transitions from such norms, physical distinctions and strong social mores challenge us. What are the trajectories of change? Why and how do they come to exist? Which changes are 'right' for us or what goals are we pursuing? These transitions in the nature of sociability and the importance of physical place serve as backdrop and shapers of the changes to work and working, and to life and living. That these changes are being sustained, magnified, and shaped by the many new uses, multiplicity and expansion of new media, challenge us to consider the nature of ethical activity regarding social behavior and ICTs.

The four papers that make up this special issue extend from presentations given at a workshop on "Digital Culture: New Forms of Living and Organizing?" which was held in June 2008 and hosted by the University of Salford. The workshop brought together more than 20 presenters and 60 attendees with a goal to better understand the conceptual, empirical and phenomenological aspects of life that blurs so called online and physical realities ${ }^{3}$ with equally indistinct boundaries regarding work and life.

\footnotetext{
${ }^{1}$ The putting out system was essentially a form of outsourcing to the home to facilitate the production of various products including textiles.

${ }^{2}$ Of course we acknowledge people might have friends at work, we merely wish to point out the fact that disconnects between particular places and things can occur - not all your friends may be people you work with for example.

${ }^{3}$ It is arguable that distinctions between 'online' and 'offline' are increasingly problematical.
} 
In our call for papers we noted that access to digital technologies remains variable but it is clear their presence and uses are increasingly important features of contemporary life. We further noted that more broadly, digital technologies are merging into physical infrastructures - at home, in transport, at work and school, and even walking 'alone' while texting - and this suggests that these play ever-more important roles in helping us construct and carry our identity and our places in an evolving society. Our call, and the focus of many papers at the workshop, emphasized that in conceptualizing our lives in digital culture terms we demonstrate the vibrancy of the scope for research of ICTs, organizations and society. Moreover, such a conceptualization challenges common notions that portray the broad field of computing and ICTs as struggling and; in particular, that ICT usage in organizations is about managerial control or industrial age models of economic production. Consequently, scholars of computing, media, organization, and society are each able to contribute to this burgeoning sociotechnical transformation bringing complimentary skills, perspectives and insights. Given the focus, we attracted scholars and experts from a range of complementary intellectual communities.

Topics covered at the workshop related to: community regeneration, community reporting, internet enabled networked organisations, the commodification of community, corporate and mass media effects upon football fans, public/private considerations in a social media saturated world, the social consequences of wireless technology, digital games and media convergence, impacts of ICTs in the estate agency sector of the United States, police blogs and shop blogs. The discourse across the workshop highlighted a range of social and ethical implications. Scholarly discourse is often at its best in raising issues and framing them in useful ways for others. We found ourselves considering difficult issues of conduct and responsibility. Many papers challenged notions of actions and behaviors that are emerging around digital presentation of self, proper actions online, and the nature of working in mixed digital and physical environments. Overall, the workshop made it clear that ICTs are increasingly becoming infused with our personal and public lives, they are shaping how we experience it and we are experiencing a variety of difficulties (and opportunities) because of this. Thus, it appears necessary to engage further with programmes of education (formal and informal) regarding the role and capabilities of ICTs in our everyday experiences in order to enable the population to engage with them in an informed way if they so choose.

Drawing from the work presented at this workshop, we encouraged a number of authors to advance their work towards this special issue. We asked these authors to develop papers where the focus of attention and discussion are the ethical and social implications of the work they presented. The four papers that follow bring together our interest in digital cultures relative to these aspects. Each of the papers considers aspects of how work and the others parts of our lives are blurring even as that which is physical is increasingly bound-up with virtual aspects of life and work.

We open the collection of papers with one that focuses upon a very personal part of our lives. Hales' analysis sheds light on how people are using various ICTs in support of their romantic relations. She draws on the concept of relationship maintenance and illustrates how ICT are used in sustaining romantic relations. Her findings suggest that the uses of ICT do not alter relationship behaviors, serving primarily as another channel for support. She also highlights that, where used, the ethical deployment of computer mediated communication tools is a key factor in successful relationship maintenance. Hales' work suggests a need for further work into ICT mediated romantic relationships in terms of the forms of media deployed and the ethical dynamics as they unfold over time particularly as related to online relationship termination.

More broadly, Richardson and Hessey's paper also focuses on relationships and the nature of online social networking sites. Drawing from interviews and focus groups of usage they articulate online social relations as both robust and as likely to magnify the uses of other connective technologies. That is, rather than thin connections via a single platform; online social activity occurs across multiple channels and reaches a socially satisfying level of interaction. Like earlier work their findings suggest that Facebook, a particular form of social networking site seems to be used for sustaining existing relationships, but not for making new connections. However, beyond this, and they identify Facebook as a relationship archiving facility. They nuance our understanding of the role of 'mass 
friending' in such spaces beyond discourses of identity work. Friending is not just about saying something about us; it is about providing mechanism for developing a form of autobiographical record. This raises some interesting implications - not least, what happens to the biography if Facebook dissolves? Thus, what moral obligation does Facebook have towards its users to provide a long-lived service given it has, and continues, to use their data for commercial purposes? Should a 'port my Facebook biography' application be provided?

Moving further to consider commercial considerations, Fletcher and Greenhill's work focuses on the emergent entrepreneurial activity of online 'blogshops' among teens. They argue that blogshops raise ethical issues regarding the presentation of the female teenage owners' 'self' to others and the relationship they maintain with consumers and other blogshop owners. These blogshops arose from the desire of teen girls interested in sharing, and then selling, fashion or trendy clothing. The blogshops build out as virtual stores presented in the social media of blogging. They have physical manifestations of inventory and sales/delivery and increasingly sophisticated mixes of online stores and conversations around the items and trends presented for sale in these stores. This mix of digital and physical culture, of buying, selling and talking, and the ways in which online in real places are mixed together illustrates what seems blurring to many and clear to these young teens.

In the closing paper McLean and Wainwright are very much concerned with the public sphere. They deploy critical social theory to conceptualize the role of commercial interests in online social life. Their empirical setting, English football, is a passionate and popular form of socializing and entertainment for many. This, in turn, draws commercial interests who draw on this popularity and passion for corporate interest. They shed light on how smart and motivated people from all sides engage in control of the social forum, leading to an extended and useful reflection on the ethical aspects of institutional and individual interests and the realization of corporate goals across physical and virtual spaces. Perhaps most fundamentally, they suggest that far from being empowered, supporters remain marginal in the trajectory of football's development, despite their significant investments.

Together, these four papers also suggest the broad scope and far-reaching implications of the blurred world of the early $21^{\text {st }}$ century. Due in some part to the rapid and expansive take up of ICT, our concepts and practices of work and play, the ways in which we relate and connect to family and others, and our reliance on traditional physical and social boundaries, are blurring in ways that reflect struggle, innovation, new forms of sociability and longstanding social norms. What fun.

\section{Biographies:}

Ben Light is Professor of Technology and Society within the School of Media, Music and Performance at the University of Salford and Director of the IS, Organisations and Society Research Centre. His research concerns the appropriation of configurable technologies within work, organisations and society. Digital Games and Social Media are central to his current research where he has, and continues to, undertake research regarding social networking sites (Facebook, YouTube, Gaydar) and the Sony PS3 SingStar game. He has published in journals such as Information Technology and People, Communications of the ACM, New Technology, Work and Employment, and the Journal of Information Technology. 